PROCEEDINGS OF THE

AMERICAN MATHEMATICAL SOCIETY

Volume 129, Number 9, Pages 2803-2807

S 0002-9939(01)05861-0

Article electronically published on February 9, 2001

\title{
AN ADDITION THEOREM FOR THE COLOR NUMBER
}

\author{
JAN M. AARTS AND ROBBERT J. FOKKINK \\ (Communicated by Alan Dow)
}

\begin{abstract}
There is a close relation between the color number of a continuous map $f: X \rightarrow X$ without fixed points and the topological dimension. If $f$ is an involution, the color number is also related to the co-index. An addition theorem for the color number is established thus underscoring the interrelations between color number, dimension and co-index.
\end{abstract}

\section{INTRODUCTION}

Our main result is an addition theorem for the color number of maps that is similar to the addition theorem of dimension theory. All topological spaces under consideration are metrizable. All mappings are assumed be continuous.

Definition 1. Suppose that $f: X \rightarrow X$ is a map of a topological space to itself. A subset $B$ of $X$ is called a color of the map $f$ if $f^{-1}(B) \cap B=\emptyset$ or, equivalently, $f(B) \cap B=\emptyset$. A coloring of $f$ is a cover of $X$ consisting of colors of $f$.

Note that if $B$ is a color of $f$, then so is $f^{-1}(B)$. It is known that every map has a coloring consisting of three colors; see 8 and 6 . The situation is totally different if we consider colorings consisting of open or closed sets only. As usual, an open coloring is a coloring consisting of open colors.

Definition 2. Suppose that $f: X \rightarrow X$ is a fixed-point free map. The color number $C(f)$ is the minimal cardinality of an open coloring of $f$.

Note that the color number $C(f)$ may not be finite. We then write $C(f)=$ $\infty$. If, for example, $\alpha$ is the antipodal map on the unit sphere in Hilbert space, then $C(\alpha)=\infty$. A less elementary example, due to Mazur, is related to the Galvin-Prikry lemma from infinite combinatorics [8, Theorem 3.4]. By contrast, closed maps with bounded fibers on a finite-dimensional space have a finite color number [4]. For a fixed-point free homeomorphism $f$ of an $n$-dimensional space we have $C(f) \leq n+3$. If moreover $f$ is an involution, then $C(f) \leq n+2$; see [1]. The color number of the antipodal map of the $n$-dimensional sphere is equal to $n+2$.

Suppose that $f: X \rightarrow X$ is a fixed-point free map. We say that a subset $A$ of $X$ is invariant if $f(A) \subset A$. For an invariant subset $A$ of $X$ the restriction $f: A \rightarrow A$ is denoted by $f_{A}$. It is to be noted that a color of $f_{A}$ is a subset of $A$. The following addition theorem is our main result.

Received by the editors November 9, 1999 and, in revised form, January 15, 2000.

2000 Mathematics Subject Classification. Primary 54F45, 55M10.

Key words and phrases. Color number, dimension, co-index, involution.

(C)2001 American Mathematical Society 
Theorem 1. Suppose that $X$ is a metric space. Let $f: X \rightarrow X$ be a fixed-point free map such that $X=A \cup B$ for invariant subsets $A$ and $B$ of $X$. Then $C(f) \leq$ $C\left(f_{A}\right)+C\left(f_{B}\right)-1$.

We will prove this theorem in the next section. The relation between the color number and the co-index will be discussed in section 3 .

\section{Proof OF THE MAIN RESUlt}

The following simple lemma will be very useful.

Lemma 1. Let $f: X \rightarrow X$ be a fixed-point free map and suppose that $A$ is an invariant subspace. Suppose that the subset $C$ of the subspace $A$ is a closed color of $f_{A}$. Then there exists an open subset $W$ of $X$ such that $f_{A}^{-1}(C) \subset W$ and $W$ is a color of $f$.

Proof. The colors $C$ and $f_{A}^{-1}(C)$ are disjoint closed subsets of the subspace $A$. Hence, these colors are separated subsets of $X$. It follows that there are disjoint open subsets $U$ and $V$ of $X$ such that $f_{A}^{-1}(C) \subset U$ and $C \subset V$. Define $W=$ $U \cap f^{-1}(V)$. One readily verifies that $W$ is an open color which contains $f_{A}^{-1}(C)$.

Corollary 1. Suppose that $f: X \rightarrow X$ is a fixed-point free map and that $\mathcal{C}$ is a collection of $n$ colors of $f$ each of which is open or closed. Then there exists an open coloring of $f$ with cardinality at most $n$.

Proof. Taking $X=A$ in Lemma 1 we can replace each closed color $B$ in the coloring $f^{-1}(\mathcal{C})$ of $f$ by an open color $U$ such that $B \subset U$.

From the corollary it follows that the color number of a map $f: X \rightarrow X$ is the minimal cardinality of a cover of $X$ consisting of colors that are open or closed. The following lemma relates an open coloring of an invariant subspace to a collection of open colors of the space.

Lemma 2. Suppose that $f: X \rightarrow X$ is a fixed-point free map and that the subset $A$ of $X$ is an invariant subspace. Let $\mathcal{U}$ be an open coloring of $f_{A}$ consisting of $m$ colors, $m \in N$. Then there exists a collection $\mathcal{V}$ consisting of at most $m$ open colors of $f$ such that $A \subset \cup \mathcal{V}$.

Proof. In a standard fashion, in the subspace $A$ there is a closed shrinking $\mathcal{G}$ of $\mathcal{U}$. Then $\mathcal{G}$ is a closed coloring of $f_{A}$ and so is the collection $\mathcal{H}=f_{A}^{-1}(\mathcal{G})$. By Lemma! for each member $H$ of $\mathcal{H}$ there is an open color $V$ of $f$ such that $H \subset V$. Let $\mathcal{V}$ be the collection of all $V$ obtained in this way.

Proof of the main theorem. We may assume that both $f_{A}$ and $f_{B}$ have a finite color number, say $n=C\left(f_{A}\right)$ and $m=C\left(f_{B}\right)$. By Lemma 2, there exist families $\mathcal{U}=\left\{U_{i} \mid i=1, \ldots, n\right\}$ and $\mathcal{V}=\left\{V_{j} \mid j=1, \ldots, m\right\}$ of open colors of $f$ such that $\mathcal{U}$ covers $A$ and $\mathcal{V}$ covers $B$. The union $\mathcal{V} \cup \mathcal{W}$ is a coloring of $f$ with $n+m$ colors.

To complete the proof we remove one of the colors so as to obtain the required coloring of $f$. Consider the collection of open sets $\mathcal{W}=\left\{U_{i} \mid i=1, \ldots, n-1\right\} \cup$ $\left\{V_{j} \mid j=1, \ldots, m-1\right\}$. Define $R=X \backslash \bigcup \mathcal{W}$. We claim that $R$ is a closed color of $f$. To show that $R$ is a color we prove $f^{-1}(R) \cap R=\emptyset$. Let $y \in f^{-1}(R)$. Without loss of generality we may assume that $y \in A$. Since $A$ is invariant, $f(y) \in A$. As $f(y) \in R$, we have $f(y) \in U_{n}$. Since $U_{n}$ is a color, $y \notin U_{n}$, whence $y \in U_{i}$ for some $i<n$. It follows that $y \notin R$, whence $f^{-1}(R) \cap R=\emptyset$. This proves the claim. 
The collection $\mathcal{W} \cup\{R\}$ is a coloring of $f$, each element of which is open or closed. According to Corollary 1 the color number of $f$ is at most $n+m-1$.

\section{Color Number AND CO-INDEX}

The antipodal map of the $n$-dimensional sphere $S_{n}$ is denoted by $\alpha_{n}$. The Borsuk-Ulam theorem states that there is no equivariant map from $\alpha_{n}$ to $\alpha_{m}$ if $n>m$. This fact has been employed by Conner and Floyd to define the co-index of a fixed-point free involution 3 .

Definition 3. The co-index $c(\tau)$ of a fixed-point free involution $\tau$ on a topological space $X$ is the minimal number $n$ such that there exists an equivariant map from $\tau$ to $\alpha_{n}$. If there exists no such map, $c(\tau)=\infty$.

Surprisingly, the color number and the co-index of a fixed-point free involution are intimately related.

Theorem 2. If $\tau: X \rightarrow X$ is a fixed-point free involution, then $C(\tau)=c(\tau)+2$.

Proof. Let $\tau: X \rightarrow X$ be a fixed-point free involution. We first show that $C(\tau) \leq$ $c(\tau)+2$. We may assume that $c(\tau)$ is finite. Thus there exists an equivariant map from $\tau$ to an antipodal map $\alpha_{n}$ on $S^{n}$ for some $n$, that is, there exists a map $f: X \rightarrow S^{n}$ such that $f \circ \tau=\alpha_{n} \circ f$. A coloring of $\alpha_{n}$ with $n+2$ colors is pulled back onto a coloring of $\tau$ to obtain a coloring of $\tau$. It follows that $C(\tau) \leq c(\tau)+2$.

To show that the opposite inequality $C(\tau) \geq c(\tau)+2$ also holds, we may assume that $C(\tau)$ is finite, say $C(\tau)=n$. Let $\left\{U_{i} \mid i=1, \ldots, n\right\}$ be an open coloring of $\tau$ and let $\left\{f_{i} \mid i=1, \ldots, n\right\}$ be a partition of unity subordinate to the open coloring. For $i=1, \ldots, n$, let $g_{i}: X \rightarrow \mathbf{R}$ be the map defined by $g_{i}(x)=f_{i}(x)-f_{i}(\tau(x))$. Using the $g_{i}$ as coordinate functions we define the map $g: X \rightarrow \mathbf{R}^{n}$. Note that $g(\tau(x))=-g(x)$. For every $x$ in $X$, the sum over the coordinates of $g(x)$ is 0 , so $g(X)$ is contained in an $(n-1)$-dimensional hyperspace $V$ of $\mathbf{R}^{n}$. Observe that $f_{i}(\tau(x))=0$ whenever $f_{i}(x)>0, x \in X$. It follows that the image $g(X)$ does not contain the origin. By dividing each $g(x)$ by its norm we get an equivariant map from $\tau$ to the antipodal map on $S^{n-2}$ (the unit sphere in $V$ ). It follows that $c(\tau) \leq C(\tau)-2$.

Conner and Floyd used the co-index to generalize the Borsuk-Ulam theorem for involutions on closed manifolds. The following addition property of the co-index now readily follows from the addition theorem of color numbers.

Corollary 2. Let $\tau: X \rightarrow X$ be a fixed-point free involution such that $X=A \cup B$ for invariant subsets $A$ and $B$ of $X$. Then $c(\tau) \leq c\left(\tau_{A}\right)+c\left(\tau_{B}\right)+1$.

This addition theorem is a slight generalization of the addition property discussed in [3], as the invariant subsets are not necessarily closed. As an application of the addition property, we show that the co-index has a decomposition property which is similar to the decomposition property of topological dimension functions [5].

Corollary 3. Suppose that $\tau: X \rightarrow X$ is a fixed-point free involution. For every $n$ in $N$ the following holds: $c(\tau) \leq n$ if and only if there exists a decomposition $X=X_{0} \cup X_{1} \cup \cdots \cup X_{n}$ into invariant subspaces $X_{i}$ such that the co-index of $\tau_{X_{i}}$ is zero for each $i$. 
Proof. If $X$ admits such a decomposition, then by repeated application of the addition property it follows that $c(\tau) \leq n$. We show that the opposite implication holds. Let $\left\{U_{i} \mid i=1, \ldots, n+2\right\}$ be an open coloring of $\tau$. Start with $X_{-1}=\emptyset$ and inductively define $X_{i}=\left(U_{i} \cup \alpha\left(U_{i}\right)\right) \backslash X_{i-1}$. Obviously $C\left(X_{i}\right)=2$, whence $c\left(X_{i}\right)=0$ for every $i$. This gives the required decomposition.

Our final corollary is known [1, but we include it here, since it is a straightforward application of the addition property.

Corollary 4. Let $n \in N$. If $\tau: X \rightarrow X$ is a fixed-point free involution on a space of dimension $\operatorname{dim} X \leq n$, then $C(\tau) \leq n+2$.

Proof. By induction. An involution on a zero-dimensional space can be colored by 2 colors $[8$. A space of $\operatorname{dim} X \leq n$ admits a decomposition $X=F \cup G$ into a zero-dimensional $F_{\sigma}$-subset $F$ and a set of $\operatorname{dim} G \leq n-1$. Then $F \cup \tau(F)$ is an invariant zero-dimensional subset. Its complement $C$ is invariant and at most $(n-1)$-dimensional, since it is contained in $G$. By the induction hypothesis the color number of $\tau_{C}$ is at most $n+1$. Now apply the addition theorem for the color number.

\section{REMARKS}

The proof of Corollary 4 can also be applied to a fixed-point free homeomorphism. If $h: X \rightarrow X$ is a homeomorphism on a $n$-dimensional space, then $X$ admits a decomposition into $n+1$ zero-dimensional, invariant sets. If in addition $h$ is fixedpoint free, then the color number of an invariant zero-dimensional subspace is at most 3. Repeated applications of the addition property then show that the color number is bounded by $2 n+3$, but this upper bound is not sharp. The sharp bound of $n+3$ is derived in [1].

Conner and Floyd's addition property for the co-index is sharp; hence so is the addition property for the color number.

A relation between the color number and the genus $g(\tau)$ of a map [7] is suggested by the proof of Corollary 3 For an involution $\tau$ we have: $c(\tau)+1=g(\tau)=C(\tau)-1$.

The example of [8, Theorem 3.4] shows that there exist maps on finite-dimensional spaces with color number $\infty$. It is natural to ask whether for every map with color number $\infty$ there is a zero-dimensional invariant subspace such that the restriction to that subspace has color number $\infty$. We are unable to resolve this question.

If a map has a finite coloring consisting of Borel sets, then the map can be colored by 3 Borel sets (cf. [8, Lemma 2.1]). The antipodal map $\alpha$ of the unit sphere $S$ in Hilbert space $\ell_{2}$ has $C(\alpha)=\infty$ but admits a coloring by 2 Borel sets, namely,

$$
A_{u}=\left\{\left\langle x_{i}\right\rangle_{i} \in S \mid x_{1}=x_{2}=\ldots=x_{k}<x_{k+1}, k \in \mathbf{N}\right\}
$$

and $A_{l}=\alpha\left(A_{u}\right)$.

A recent survey of the co-index and other invariants as well as some new results are given in [6].

\section{REFERENCES}

1. Aarts, J.M., Fokkink, R.J., Vermeer, J., Variations on a theorem of Lusternik and Schnirelman, Topology 35 (1996), 1051-1056 MR 98e:55003

2. Conner, P.E., Floyd, E.E., Fixed point free involutions and equivariant maps, Bull. Amer.Math.Soc. 66 (1960), 416-441 MR 29:613 
3. Conner, P. E., Floyd, E. E., Fixed point free involutions and equivariant maps, II, Trans. Amer. Math. Soc. 105 (1962), 222-228 MR 26:768

4. Douwen, E. van, $\beta X$ and fixed-point free maps, Top. Appl. 51 (1993), 191-195 MR 94g:54030

5. Engelking, R., Theory of dimensions, finite and infinite, Sigma Series in Pure Mathematics 10, Lemgo, 1995 MR 97j:54033

6. Hartskamp, M. van, Colorings of fixed-point free maps, Thesis, Free University, Amsterdam, 1999

7. Krasnosel'skii, M.A., On the computation of the rotation of a vectorfield on finite-dimensional spheres, Dokl. Akad. Nauk SSSR 101 (1955), 401-404

8. Krawczyk, A., Steprans, J., Continuous colorings of closed graphs, Top. Appl. 51 (1993), 13-26 MR 94e:54046

9. Mill, J. van, Easier proofs of colouring theorems, Top. Appl. 97 (1999), 155-163 MR 2000i:54066

Faculty of Mathematics, Delft University, P.O. Box 5031, 2600 GA Delft, NetherLANDS

E-mail address: j.m.aarts@its.tudelft.nl

Faculty of Mathematics, Delft University, P.O. Box 5031, 2600 GA Delft, NetherLANDS

E-mail address: r.j.fokkink@its.tudelft.nl 